\title{
Using the Web to facilitate international academic exchange
}

\author{
STEPHAN LEWANDOWSKY and CRAIG D. CLARK \\ University of Western Australia, Nedlands, Western Australia, Australia
}

\begin{abstract}
Use of the World-Wide Web among academics has grown explosively during the last few years. To date, most applications of the Web have focused on the storage and dissemination of relatively static information, in part because of the cost associated with manual collating and updating of data. This paper presents an example of a dynamic worldwide database devoted to maintaining inherently transient information: The database accepts international travel itineraries submitted by individual academic psychologists, and makes them publicly available to potential hosts on the basis of various search keys. The objective of the database (http://www.psy.uwa.edu.au/wave/) is to facilitate international exchange among academics.
\end{abstract}

The World-Wide Web arguably represents one of the more important technological advances to emerge during the past few decades: Information stored across literally millions of computers worldwide can now be searched and accessed by anyone from anywhere in the world for little or no charge. The explosive growth in Web use, in particular in the academic community, has made it the delivery system of choice for information services that affect every aspect of academic life. How research is published, how and where information related to research, teaching, and the profession is obtained, and the scope of the intellectual forum in which researchers discuss and present work are all changing as the possibilities offered by this new information medium are recognized and exploited.

The Web is already used extensively for the distribution of relatively static research information, with individual academics and research groups routinely creating homepages with links to online copies of recent papers and data. In some cases, data are presented in greater detail than is practical in print and are illustrated with audio and video as well as color graphics. Some authors have gone further, exploiting the full interactive capabilities of the Web to allow readers to participate in tutorial quasiexperiments-demonstrating the experimental procedure, displaying the stimuli, and collecting responses (e.g., http://www.phil.uni-sb.de/FR/Medienzentrum/ Grafikexperiment/Grafikexperiment.html).

There are a number of Web sites that offer authors centralized distribution points for papers in psychology (see http://psych.hanover.edu/Krantz/journal.html). These electronic journals range from informal discussion groups to internationally refereed publications such as Psycolo-

The author's mailing address is Department of Psychology, University of Western Australia, Nedlands, WA 6907, Australia (e-mail: lewan@psy.uwa.edu.au) quy (http://www.princeton.edu/ harnad/psyc. html), an interdisciplinary journal sponsored by the American Psychological Association, and Noetica (http://psy.uq. edu. au/CogPsych/Noetica/), a journal devoted to cognitive science. With a few exceptions, established print journals have yet to follow mass-market publications into subscription-based online publication, but most already publish subscription details and submission information for contributers on the Web.

Even library catalogs and database services, already accessible online through less user-friendly protocols, are establishing Web interfaces in increasing numbers. These include major archives containing thousands of social science databases (see http://ssda.anu.edu.au/) and several commercial interactive databases that offer organizational subscriptions and delivery from a remote site as an alternative to maintaining and updating on-site CDROM-based delivery systems. The Periodical Contents Index (http://pci.chadwyck.com) is a good example of what the Web has to offer academic psychologists in this area, with indexing of (so far) more than six million articles in 1,595 journals from their first issue to 1990/1991.

The abundance of available information stands in contrast to the limitations associated with information search. Current internet search engines are good at locating very specific information and individual sites that can be specified by name, but are poor at collating links to sites that contain broader categories of information. As search topics become more loosely defined, the few sites of immediate interest are very rapidly obscured by hundreds or thousands of false alarms. This problem has preserved a niche for manual indexing of web resources, perhaps the earliest service offered on the Web, and one for which need continues to grow as rapidly as the Web itself. At present the most comprehensive resource indexes produced for academic psychologists are "Cognitive and Psychological Sciences on the Internet" (http:// www-psych.stanford.edu/cogsci/) and Mental Health 
Net (http://www.cmhc.com/), the latter spanning clinical as well as academic psychology. PsychWeb (http:// www.gasou.edu/psychweb/psychweb.htm) provides a similar listing directed more specifically at psychology students.

The fact that editorial mediation and manual indexing is extremely costly and time consuming has so far prevented exploitation of one of the greatest potential uses of the Web by the academic community, namely, the centralized indexing and distribution of transient information - the Web analogue of public notices. The few examples that currently exist include the WWW Virtual Library Conference Announcements Database at http:// conferences.rpd.net/ and the Worldwide Register of Positions in Psychology (PIP) at http:/www.anu.edu.au/ psychology/PiP/pip.htm. In both cases, data are collected via e-mail (also indirectly from postings to the news. announce.conferences UseNet Newsgroup in the former case) and collated manually.

This paper introduces a third example, the Worldwide Academic Visitor Exchange (WAVE). In contrast to existing transient databases, WAVE is fully interactive and fully automated. WAVE is dedicated to supporting international collaboration by facilitating contact between traveling scientists and prospective hosts. Briefly, WAVE maintains a database of international travel itineraries submitted by individuals interested in establishing contacts with colleagues while abroad. Potential hosts are invited to search the database for visitors to their area. Contact between hosts and visitors can then be established by e-mail if desired.

Anyone in the discipline who is about to travel abroad is invited to add an itinerary to the WAVE database. It does not matter which countries and cities are being visited and for how long; the only current constraint on the service is that it is not intended for domestic travel. Travelers can specify their research interests and potential colloquium titles as part of their itinerary, with specific comments available for each intended destination. Once entered, travel itineraries are password protected to avoid frivolous alterations or deletions. Itineraries are automatically deleted from the database when the dates referenced have passed.

Anyone willing to host visitors from abroad for any academic purpose is invited to search the WAVE database for matching itineraries. The database can be searched using a city, broader geographic region, or a visitor's name as search keys. Search keys can be saved and entered into the database for future automatic notification by e-mail whenever a matching itinerary is entered. For example, potential hosts located in the Dallas area can request to be notified automatically whenever anyone from abroad visits Texas or surrounding states. Notifications include all information available in the itinerary, including research interests and potential colloquium titles. To date (November 1996), some 45 prospective hosts in 13 countries have signed up for automatic notification.

WAVE is free of charge and supported by the Department of Psychology at The University of Western Australia. WAVE is in operation now at http://www.psy.uwa. edu.au/wave/.

(Manuscript received November 19, 1996; accepted for publication December 16, 1996.) 